\title{
Características Antropométricas por Posición de Juego en Mujeres Futbolistas Chilenas de la Región de Valparaíso, Chile
}

\author{
Anthropometric Attributes by Playing Position in Chilean \\ Female Football Players From the Valparaiso Region, Chile
}

\author{
Barraza, F.; Yáñez, R.**; Báez, E.*** \& Rosales, G.****
}

BARRAZA, F.; YÁÑEZ, R.; BÁEZ, E. \& ROSALES, G. Características antropométricas por posición de juego en mujeres futbolistas chilenas de la Región de Valparaíso. Int. J. Morphol., 33(4):1225-1230, 2015.

RESUMEN: Participaron del estudio 76 mujeres futbolistas de la región de Valparaíso, las edades fluctuaron entre los 18 y 30 años. Fueron evaluados los equipos femeninos profesionales de los clubes Everton, Santiago Wanderers y San Luis de Quillota, las selecciones de la universidades de Valparaíso, Técnica Federico Santa María y Universidad Viña del Mar. Las posiciones de juego fueron distribuidas en arqueras $(n=8)$, defensas $(n=25)$, volantes $(n=24)$ y delanteras $(n=18)$. Para la evaluación de la composición corporal se utilizó el protocolo establecido por la International Society for the Advancement of Kinanthropometry. Para determinar el somatotipo de las futbolistas fue utilizado el modelo de Heath \& Carter. Se fraccionó el peso corporal total a través del método pentacompartimental diseñado por Kerr. Los resultados demuestran un somatotipo promedio mesomorfo endomorfo, la distribución por equipos evidencia diferencias significativas en el mesomorfismo entre los equipos Everton y Universidad Viña del Mar ( $<<0,014)$. Entre las posiciones arquera y delantera existieron diferencias significativas en las variables peso $(\mathrm{p}<0,030)$ y endomorfia $(\mathrm{p}<0,050)$. No existieron diferencias en el porcentaje de tejido adiposo, tejido muscular, sumatoria de pliegues y estatura por posición de juego. En lo que respecta a la distribución por posición de juego, se evidencia un somatotipo meso endomorfo en las posiciones arquera y defensa, en las posiciones volantes y delanteras se aprecia un somatotipo mesomorfo endomorfo, esto demuestra una diferenciación del somatotipo entre posiciones de juego defensivas y ofensivas encontrando un mayor predominio de la endomorfia en las posiciones defensivas en comparación con las de ataque. Según el método SANOVA se concluye que las futbolistas evaluadas en este estudio presentaron características antropométricas similares, no habiendo diferencias significativas entre equipos universitarios y profesionales, ni por posición de juego.

PALABRAS CLAVE: Perfil antropométrico; Fútbol; Somatotipo; Mujer.

\section{INTRODUCCIÓN}

Actualmente el Fútbol es uno de los deportes colectivos que más se practican alrededor del mundo, en las últimas décadas el sexo femenino se ha hecho parte importante en el desarrollo de esta disciplina, el impacto mediático que hoy en día presenta es muy elevado considerando que ya existen 135 selecciones femeninas de este deporte y que alrededor de 29 millones de mujeres de todas las edades juegan al futbol en el mundo entero. Ha sido tal la repercusión que ha tenido el Fútbol femenino que Franz A. Beckenbauer en su visita a Chile con motivo de la celebración del Mundial Femenino Sub 20, en el año 2008, afirmó que el fútbol femenino se va a acercar al de los hombres pero por aspec- tos de tipo físico nunca se van a igualar (Beckenbauer, 2008). A pesar de esto es necesario determinar el perfil antropométrico en este tipo de deportistas para determinar su real estado y nivel de composición corporal, considerando que la tendencia en la formación en el futbol, determina que las variables antropométricas sean importantes al momento de definir entre otras cosas, la posición de juego más idónea, determinando el rendimiento en un salto o en un sprint (Dey et al., 2010).

Es relevante considerar que la determinación de la morfología corporal a partir de las variables antropométricas

\footnotetext{
* Escuela de Educación, Carrera Educación Física. Universidad Viña del Mar, Viña del Mar, Chile.

** Facultad de Filosofía y Educación, Pontificia Universidad Católica de Valparaíso, Valparaíso, Chile.

**** Departamento de Deportes y Recreación, Facultad de Ciencias de la Actividad Física, Universidad de Playa Ancha, Valparaíso, Chile.

***** Centro Tecnológico de Investigación y Gestión en Actividad Física, Deporte y Salud, Quintero, Chile.
} 
es parte de la valoración rutinaria de cualquier deportista, permitiendo ubicar a este y compararlo en un deporte o puesto de juego, de tal forma que permita mejorar el rendimiento individual y/o colectivo o plantear regímenes de entrenamiento con la intención de encuadrar a los atletas en la condición más próxima a una referencia (Queiroga et al., 2008). Debido a lo señalado anteriormente, se hace imprescindible determinar las características antropométricas por posición de juego en este grupo de deportistas.

\section{MATERIAL Y MÉTODO}

Para el desarrollo de este estudio, la evaluación antropométrica se realizó durante la tarde, antes de la sesión de entrenamiento, se ejecutó con la menor ropa posible y después del vaciado urinario, en los mismos lugares donde fueron realizadas las sesiones de entrenamiento de cada equipo, previo consentimiento informado aprobado por las jugadoras evaluadas. La variables fueron evaluadas en base al protocolo de marcaje de la International Society for the Advancement of Kineatropometry (ISAK) para el procedimiento de medición de 25 variables (Drinkwater, 1984; Norton \& Olds, 1996). En el desarrollo de la evaluación se utilizó un estadiómetro portátil, una balanza digital Tanita ${ }^{\circledR}$ con precisión de 100 g, un kit antropométrico Health Performance ${ }^{\circledR}$ compuesto por los siguientes instrumentos: caliper largo, caliper corto, segmómetro retráctil, escuadra metálica, plicómetro plástico medidor de pliegues cutáneos marca SLIMGUIDE® con precisión en milímetros y una cintra métrica metálica LUKFIN®.

Con la finalidad de determinar la composición corporal, se consideró el tejido adiposo, muscular, óseo, residual y de piel, utilizando las ecuaciones propuestas por Kerr (1988). Se determinó la forma corporal de las futbolistas a través del método del somatotipo propuesto por Carter (Carter \& Heath, 1990; Carter, 2002).

En esta investigación se evaluaron 3 variables básicas, peso $(\mathrm{kg})$, estatura de pie $(\mathrm{cm})$ y estatura sentado $(\mathrm{cm})$; 6 diámetros óseos (cm); Biacromial, Tórax Transverso, Tórax Anteroposterior, Biiliocrestidio, Humeral y Femoral. 10 perímetros (cm); Cabeza, Brazo relajado, Brazo contraído, Antebrazo, Tórax, Cintura, Cadera, Muslo Máximo, Muslo medio y Pierna medial. Seis pliegues cutáneos (mm); Tricipital, Subescapular, Supraespinal, Abdominal, Muslo Medio y Pierna Medial. La evaluación se realizo en posición anatómica con un orden de superior a inferior. A partir de los resultados obtenidos se determino el somatotipo, el porcentaje de masa muscular y el porcentaje de tejido adiposo entre otras variables.
Para el análisis de los datos se utilizaron los Softwares Somatotype Calculation and Analysis Software versión 1.1 (San Diego, CA, USA) y Gradhpad 6.0 (San Diego, CA, USA). Se utilizó el estadístico de Kolgomorov-Smirnov el cual entregó una distribución normal de la muestra evaluada ( $\mathrm{p}>0,05)$. Se utilizó el análisis especial de la varianza SANOVA de una vía para determinar diferencias significativas entre instituciones y por posición de juego, se estableció como valor significativo un $\mathrm{p}<0,05$. Se analizó la media actitudinal del somatotipo (SAM) basándose en las ecuaciones propuestas para determinar la homogeneidad de la muestra (Carter, 2002).

\section{RESULTADOS}

El análisis se detalla y organiza de manera que otorgue una perspectiva precisa y concreta de los resultados obtenidos. A continuación se presentan los resultados obtenidos en el estudio.

Para otorgar validez interna al estudio, se determinó el grado de acuerdo a través del análisis del coeficiente de correlación intraclase (CCI) y el nivel de consistencia medido a través del índice de correlación de Pearson para las variables peso estructurado y peso real. Se evidenció una correlación intraclase para medidas individuales de 0,827 , una correlación intraclase para medidas promedio de 0,905 y un nivel de correlación de Pearson de 0,869. Se apreció para los tres estadísticos un nivel de significancia $\mathrm{p}<0,01$.

En relación al análisis de los equipos de Fútbol Femenino, en los aspectos relacionados con los componentes de la composición corporal y el somatotipo sólo se encontraron diferencias significativas en el componente mesomorfia entre los equipos de la Universidad de Viña del Mar y el club Everton $(<0,014)$. En las otras variables de estudio no se encontraron diferencias estadísticamente significativas entre los equipos evaluados. Considerando la determinación del somatotipo de manera general e integrada evaluada a través de SANOVA, no se evidenciaron diferencias significativas entre las instituciones evaluadas $(\mathrm{p}=0,157)$ (Tabla I). Los resultados obtenidos en la determinación de la media actitudinal del somatotipo (SAM) muestran que el equipo de San Luis de Quillota $(1,47 \pm 0,53)$ es el que encontraron una mayor homogeneidad de los datos obtenidos, le siguen Santiago Wanderers $(1,57 \pm 0,57)$, UVM $(1,59 \pm 0,90)$, UV $(1,59 \pm 1,07)$, UTFSM $(1,73 \pm 0,86)$ y Everton $(2,01 \pm 0,97)$.

En lo que concierne a los resultados obtenidos por posición de juego, se aprecia que las jugadoras más altas son las arqueras, le siguen las defensas, luego las volantes y finalmente las delanteras (Tabla II). En la determinación del 
somatotipo, las arqueras presentan un somatotipo meso endomorfo $(5,0-4,1-1,6)$, las jugadoras que se desempeñan como defensas un somatotipo meso endomorfo $(4,6-3,7-1.8)$, las volantes un somatotipo mesomorfo endomorfo $(4,4-4,0-$ 1.6) y las delanteras un somatotipo mesomorfo endomorfo $(3,6-3,6-1,9)$. El somatotipo promedio del grupo de futbolistas evaluadas fue determinado como mesomorfo endomorfo $(4,4-$ $3,9-1,8)$. Se evidenciaron diferencias significativas en las variables peso corporal $(\mathrm{p}=0,030)$ y componente endomorfia $(p=0,018)$ entre las posiciones arquera y delantera. No se encontraron diferencias significativas por posición de juego en las variables masa adiposa, masa muscular y sumatoria de 6 pliegues $(\mathrm{p}>0,05)$. Considerando la determinación del somatotipo de manera general e integrada evaluada a través de SANOVA, no se evidenciaron diferencias significativas entre las instituciones evaluadas $(\mathrm{p}=0,153)$. Los resultados obtenidos en la determinación de la media actitudinal del somatotipo (SAM) evidencian que la posición delanteras $(1,5 \pm 0,60)$ es la que presenta una mayor homogeneidad de los datos obtenidos, le siguen las volantes $(1,60 \pm 0,77)$, defensas $(1,80 \pm 0,83)$ y las arqueras $(2,1 \pm 1,29)$.
Los resultados presentes en la Figura 1 representan la distribución y el promedio de la muestra según equipo de juego. La Figura 2 representa la distribución y el promedio de las futbolistas distribuidas por su posición en el campo de juego.

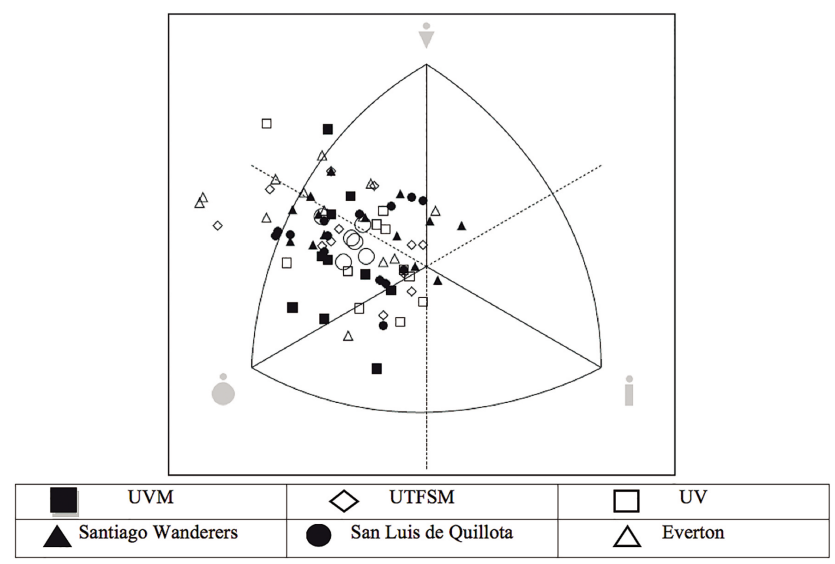

Fig. 1. Distribución de los puntos en la somatocarta y promedios por equipo.

Tabla I. Promedios y desviaciones estándar de las variables antropométricas evaluadas distribuidas por equipo.

\begin{tabular}{|c|c|c|c|c|c|c|c|c|c|c|c|c|}
\hline & \multicolumn{2}{|c|}{ UV(a) } & \multicolumn{2}{|c|}{ UTFSM(b) } & \multicolumn{2}{|c|}{ UVM(c) } & \multicolumn{2}{|c|}{ Everton(d) } & \multicolumn{2}{|c|}{ S. Wanderers(e) } & \multicolumn{2}{|c|}{ San Luis(f) } \\
\hline & \multicolumn{2}{|c|}{$n=12$} & \multicolumn{2}{|c|}{$n=12$} & \multicolumn{2}{|c|}{$n=11$} & \multicolumn{2}{|c|}{$n=13$} & \multicolumn{2}{|c|}{$n=14$} & \multicolumn{2}{|c|}{$n=14$} \\
\hline & Media & DE & Media & DE & Media & DE & Media & DE & Media & DE & Media & DE \\
\hline Peso (kg) & 59,7 & 5,9 & 56,1 & 6,5 & 60,2 & 5,6 & 61,2 & 11,4 & 59,3 & 9,8 & 57,0 & 5,6 \\
\hline Talla (cm) & 162,4 & 5,9 & 157,9 & 6,8 & 161,9 & 6,1 & 159,3 & 7,2 & 161,5 & 7,6 & 159,7 & 5,8 \\
\hline \% Masa Adiposa & 30,8 & 2,7 & 32,5 & 4,4 & 33,1 & 3,8 & 28,7 & 5,0 & 29,9 & 3,9 & 30,9 & 4,6 \\
\hline \% Masa Muscular & 41,7 & 3,1 & 41,8 & 3,2 & 40,2 & 4,6 & 44,6 & 3,6 & 42,7 & 3,3 & 42,4 & 3,8 \\
\hline$\sum 6$ Pliegues & 81,3 & 28,3 & 97,7 & 29,5 & 93,3 & 18,1 & 90,1 & 36,7 & 87,9 & 25,8 & 86,6 & 20,3 \\
\hline Endo & 4,0 & 1,1 & 4,4 & 1,4 & 4,3 & 0,9 & 4,9 & 1,7 & 4,0 & 1,3 & 4,3 & 1,2 \\
\hline Meso & 3,3 & 1,4 & 3,9 & 1,1 & $3,1 *$ & 1,5 & $4,6^{*}$ & 1,2 & 4,1 & 0,8 & 3,8 & 0,7 \\
\hline Ecto & 1,9 & 0,9 & 1,8 & 1,0 & 1,7 & 0,7 & 1,4 & 1,0 & 1,8 & 0,9 & 1,8 & 0,8 \\
\hline SAM & 1,59 & 1,07 & 1,73 & 0,86 & 1,59 & 0,90 & 2,01 & 0,97 & 1,57 & 0,57 & 1,47 & 0,53 \\
\hline
\end{tabular}

Diferencias significativas en prueba de comparaciones SANOVA entre los grupos c y d*(p<0,014).

Tabla II. Promedios y desviaciones estándar de los sujetos de evaluados por posición de juego.

\begin{tabular}{lcccccccccc}
\hline & \multicolumn{2}{c}{ Arqueras(a) } & \multicolumn{2}{c}{ Defensas(b) } & \multicolumn{2}{c}{ Volantes(c) } & \multicolumn{2}{c}{ Delanteras(d) } & \multicolumn{2}{c}{ Promedio } \\
\cline { 2 - 10 } & \multicolumn{2}{c}{$\mathbf{n = 8}$} & \multicolumn{2}{c}{$\mathbf{n = 2 6}$} & \multicolumn{2}{c}{$\mathbf{n = 2 4}$} & \multicolumn{2}{c}{$\mathbf{n = 1 8}$} & \multicolumn{2}{c}{$\mathbf{n = 7 6}$} \\
& Media & $\mathbf{D E}$ & Media & $\mathbf{D E}$ & Media & $\mathbf{D E}$ & Media & DE & Media & DE \\
\hline Peso (kg) & $65,8^{*}$ & 12,6 & 59,1 & 7,4 & 58,6 & 6,0 & $55,9^{*}$ & 6,7 & 59,0 & 7,7 \\
Talla (cm) & 163,3 & 4,9 & 161,3 & 7,0 & 159,8 & 6,4 & 158,8 & 6,8 & 160,5 & 6,6 \\
\% Masa Adiposa & 31,2 & 4,2 & 32,1 & 3,8 & 30,8 & 4,6 & 29,3 & 4,4 & 30,9 & 4,3 \\
\% Masa Muscular & 41,9 & 3,8 & 41,3 & 3,9 & 42,5 & 3,7 & 43,5 & 3,6 & 42,3 & 3,8 \\
I 6 Pliegues & 104,2 & 36,2 & 90,5 & 25,6 & 92,1 & 26,3 & 77,4 & 20,8 & 89,6 & 26,6 \\
Endo & $5,0 * *$ & 1,9 & 4,6 & 1,1 & 4,4 & 1,3 & $3,6 * *$ & 1,0 & 4,4 & 1,3 \\
Meso & 4,1 & 1,4 & 3,7 & 1,4 & 4,0 & 1,0 & 3,6 & 1,1 & 3,9 & 1,2 \\
Ecto & 1,6 & 1,0 & 1,8 & 0,9 & 1,6 & 0,8 & 1,9 & 0,7 & 1,8 & 0,9 \\
SAM & 2,1 & 1,29 & 1,8 & 0,83 & 1,6 & 0,77 & 1,5 & 0,6 & 1,7 & 0,87 \\
\hline
\end{tabular}

Diferencias significativas en prueba de comparaciones SANOVA entre los grupos a y d*(p<0,030), ** $(\mathrm{p}<0,018)$. 


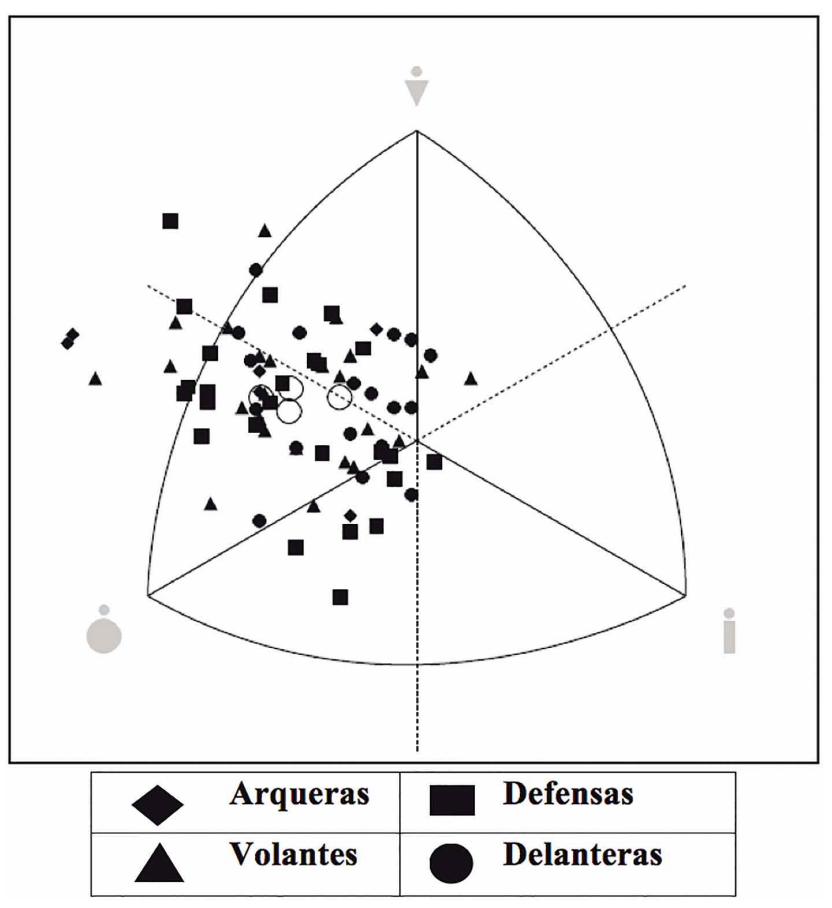

Fig. 2. Distribución de los puntos en la somatocarta y promedios, por posición de juego.

\section{DISCUSIÓN}

Es importante considerar las variaciones en la composición corporal que las jugadoras de Fútbol puedan presentar en relación a la posición de juego en la que se desempeñan, en este aspecto la evaluación cineantropométrica permite generar criterios para la selección de talentos y distribución de las deportistas considerando la especialización en el campo de juego con la finalidad de conseguir su mayor performance deportiva. Es por esto que se sugiere considerar la función dentro del campo de juego a la hora de interpretar la composición corporal (Carling \& Orhant, 2010).

Los resultados de este estudio evidencian un somatotipo promedio mesomorfo endomorfo lo que se corrobora con los resultados obtenidos por Can et al. (2004), en el cual las mujeres futbolistas evaluadas presentaron un somatotipo promedio de 3,1-3,6-2,4 (mesomorfo endomorfo). En este trabajo de investigación en general no se evidenciaron diferencias antropométricas significativas por posición de juego, al respecto, un estudio realizado con 24 jugadoras croatas de élite evidenció que no existían diferencias morfológicas significativas entre las jugadoras en función de la posición que ocupaban dentro del sistema de juego, diferenciando entre las guardametas, las defensas, las centrocampistas y las delanteras (Juric et al., 2007). Evidencias que permiten señalar que actualmente, en el caso de las mujeres futbolistas evaluadas en este estudio, no existe diferenciación en cuanto a las características antropométricas por posición en el campo de juego. Aún así los resultados muestran diferente somatotipo entre las posiciones de ataque y defensa, predominando en las posiciones ofensivas el componente muscular en comparación con las posiciones defensivas.

En lo que concierne a las variables tejido adiposo, peso y talla se aprecia que en el grupo de futbolistas chilenas sólo se encontraron diferencias significativas entre el peso de las arqueras y delanteras, en las demás variables no se evidencian diferencias significativas por posición de juego ni por equipos. Al respecto un estudio confirmó la existencia de diferencias estadísticamente significativas entre mujeres futbolistas inglesas de nivel internacional y aquellas que participan en competiciones de ámbito regional, siendo estas últimas las que acumulan mayor porcentaje de grasa. En lo que hace referencia a la talla, se constata que las jugadoras más altas son las defensas centrales, seguidas de porteras, delanteras, y centrocampistas, siendo las laterales las que menor altura registran (Todd et al., 2002). Con relación a lo señalado, es preciso mencionar que en este estudio la estatura más elevada la obtuvieron las arqueras seguidas por las defensas, volantes y finalmente delanteras, esto deja de manifiesto que las jugadoras que se desempeñan en las posiciones de la zona defensiva presentan valores más altos en la estatura en comparación con las posiciones de la ofensiva, aspecto que puede ser utilizado como un criterio para la selección de talentos esta tendencia se puede apreciar incluso en futbolistas del sexo masculino (Henríquez-Olguín et al., 2013). Aún así los estudios realizados con jugadoras de fútbol y la heterogeneidad encontrada sobre las características de las futbolistas sugieren que, quizás no existan unas características específicas de la mujer futbolista, de la misma manera que ocurre en los hombres futbolistas (Casajús, 2004), esto se confirma con los diversos estudios que se han realizado con relación al somatotipo de las mujeres futbolistas, se han publicado diversos valores en fútbol femenino, siendo consideradas en fútbol de salón como mesoendomorfas (Queiroga et al.; Levandosky et al., 2007), en fútbol convencional se han establecido como endomorfas mesomorfas (Can et al.), un estudio realizado en futbolistas chilenas demostró que la clasificación del somatotipo, de acuerdo a la forma de ambos grupos al ser estadísticamente homogéneo, fue clasificado como mesoendomórfica, con valores de 3,9-4,5-1,6 para endo, meso y ecto, respectivamente (Almagiá Flores et al., 2008). Como se puede apreciar todos los estudios revisados muestran distintos somatotipos promedio, lo que demuestra una gran dispersión de los perfiles en este grupo de deportistas. 
Es importante considerar que el exceso de tejido adiposo influye negativamente en el rendimiento deportivo, puesto que el aumento del peso corporal derivado de la grasa no se acompaña de un incremento de la capacidad para producir mayor fuerza. Considerando que la aceleración es directamente proporcional a la fuerza, pero inversamente proporcional a la masa corporal, el exceso de tejido adiposo a un nivel dado de fuerza aplicada, resultara en cambios más lentos en la velocidad y en la dirección, y este exceso de adiposidad también incrementa el costo metabólico de actividades físicas que implican el movimiento de la masa corporal (Shepard \& Astrand, 1998). El grado de adiposidad tiene más influencia en el rendimiento que el peso corporal total. En general cuando mayor es la tejido adiposo total, peor es el rendimiento deportivo en actividades como el Futbol (Willmore \& Costill, 2004).

Es relevante señalar que los estudios realizados en mujeres futbolistas muestran resultados no concluyentes. Los estudios realizados con jugadoras de fútbol y la heterogeneidad encontrada sobre las características de las futbolistas sugieren que, quizás no existan unas características específicas de la mujer futbolistas (Casajús). Incluso, estas características podrían ser diferentes según la demarcación que ocupase cada jugadora y las funciones que desarrollase dentro del sistema de juego de su equipo. En este estudio las arqueras y defensas presentaron un somatotipo meso endomorfo, mientras que las volantes y delanteras presentaron un somatotipo mesomorfo endomorfo, resultado que de alguna manera divide el somatotipo entre posiciones defensivas y posiciones ofensivas. A partir de esto podemos señalar que las futbolistas de las posiciones defensivas presentaron un mayor predomino de la endomorfia con relación a los demás componentes del somatotipo, en el caso de las jugadoras de las posiciones ofensivas se pudo determinar que la endomorfia y la mesomorfia presentaron valores similares, por ende las jugadoras de ataque presentaron una mejor relación entre el tejido muscular y adiposo, a partir de esto es importante considerar los componentes de la composición corporal en la evaluación de deportistas de alto nivel ya que esto permite cuantificar las masas plásticas, adiposa y muscular, facilita cuantificar el peso extra o de lastre y su relación con la eficiencia o rendimiento mecánico, ayuda a interpretar mejor los efectos anabólicos del entrenamiento físico o ejercicio, su relación con el coste metabólico, la producción de fuerza, la capacidad de trabajo físico y el rendimiento muscular (Berral de la Rosa et al., 2010). Diversos estudios han demostrado que las mujeres deportistas, generalmente, tienen un 5-10\% más de grasa relativa que los hombres, aún así, es deseable competir con un cuerpo con baja grasa relativa, pues este tejido actúa como peso muerto, limitando la realización de acciones rápidas (Queiroga et al.), en este aspecto la relación del componen- te antropométrico con la capacidad física de las deportistas le otorga un real valor a la determinación del perfil antropométrico en esta población.

En los próximos años es de esperar una mayor expansión del fútbol femenino así como una mejora de los datos antropométricos, físicos, fisiológicos, técnicos, tácti$\cos$ y todos aquellos que hacen referencia a las características de las jugadoras así como a su rendimiento en competición, de manera paralela al auge y desarrollo del fútbol femenino (Hoare \& Warr, 2000; Gorostiaga, 2002; Tejedor \& Torés, 2003).

\section{AGRADECIMIENTOS}

A los clubes de Fútbol profesional y universitario que participaron de la investigación. A las jugadoras, entrenadores y el cuerpo técnico de cada uno de los equipos, sin ellos este trabajo no hubiese visto la luz. Agradecemos las ganas de cada grupo de seguir incentivando la práctica del Fútbol en nuestro país, sin duda el trabajo que realizan cada día permite el progreso del Fútbol femenino en Chile.

BARRAZA, F.; YÁÑEZ, R.; BÁEZ, E. \& ROSALES, G. Anthropometric attributes by playing position in Chilean female football players from the Valparaiso Region. Int. J. Morphol., 33(4):1225-1230, 2015.

SUMMARY: Seventy-six female football players from the Valparaiso Region. Players'age fluctuated between 18 and 30 years participated in this research. Female professional teams from Everton, Santiago Wanderers and San Luis de Quillota clubs, as well as the teams from Federico Santa María Technical University and Viña del Mar University were evaluated. Playing positions were: goalkeepers $(n=8)$, defenders $(n=25)$, attacking midfielders $(n=24)$ and strikers $(n=18)$. To measure body composition the protocol established by the International Society for the Advancement of Kinanthropometry was used, according to the restricted profile described by Drinkwater and Norton \& Olds. The Heath and Carter method was used to determine the somatotype of the female football players. Total body mass was divided using Kerr's Five-Way Fractionation method. Results show an average somatotype of mesomorph and endomorph. In the distribution by teams, the results showed significant differences of mesomorph between the Everton Club and the Viña del Mar University $(p<0.014)$. Between the goalkeepers and the strikers there were significant differences regarding weight $(\mathrm{p}<0.030)$ and endomorph $(\mathrm{p}<0.050)$. No differences in the percentage of adipose tissue, muscle tissue, the sum of folds and height in relation to playing position were found. Regarding the distribution by playing position, the results showed a meso-endomorph somatotype for the goalkeepers and defenders, and a mesomorph endomorph somatotype for the attacking midfielders and strikers. This reveals 
a differentiation of somatotypes between offensive and defensive playing positions, finding a higher prevalence of the endomorph somatotype in defensive playing positions compared to the attack positions. Using the SANOVA method, the study concludes that the anthropometric attributes of the female football players evaluated are similar, with no significant differences between university teams and professional teams or by playing position.

KEY WORDS: Anthropometric profile; Football; Somatotype; Female.

\section{REFERENCIAS BIBLIOGRÁFICAS}

Almagiá Flores, A. A.; Rodríguez Rodríguez, F.; Barraza Gómez, F. O.; Lizana Arce, P. J. \& Jorquera Aguilera, C. A. Anthropometric profile of female football - Soccer chilean players. Int. J. Morphol., 26(4):817-21, 2008.

Beckenbauer: "Vidal se ha ganado el respeto del público alemán" (18 de Noviembre de 2008). En: Diario La Tercera, Sección Fútbol. Santiago de Chile, La Tercera, 2008. Disponible en: h t t p : / / w w w. 1 a t e r cera.c $1 /$ con te n i do/82_75220_9.shtml

Berral de la Rosa, F. J.; Rodríguez-Bies, E. C.; Berral de la Rosa, C. J.; Rojano Ortega, D. \& Lara Padilla, E. Comparison of anthropometric equations for estimation muscle mass in badminton player. Int. J. Morphol., 28(3):803-10, 2010.

Can, E.; Yilmaz, I. \& Erden, Z. Morphological characteristics and performance variables of women soccer players. J. Strength Cond. Res., 18(3):480-5, 2004.

Carling, C. \& Orhant, E. Variation in body composition in professional soccer players: interseasonal and intraseasonal changes and the effects of exposure time and player position. J. Strength Cond. Res., 24(5):1332-9, 2010.

Carter, J. E. L. \& Heath, B. H. Somatotyping - Development and applications. Cambridge studies in biological anthropology. Cambridge, University Press, 1990.

Carter, J. E. L. The Heath-Carter Somatotype method. San Diego, San Diego State University Syllabus Service, 2002.

Casajús, J. A. Perfil fisiológico del jugador de fútbol. Madrid, IV Congreso Internacional de las Ciencias del Deporte del Real Madrid C. F., 2004.

Dey, S. K.; Kar, N. \& Debray, P. Anthropometric, motor ability and physiological profiles of Indian national club footballers: a comparative study. S. Afr. J. Res. Sport Phys. Educ. Recreat., 32(1):43-56, 2010.

Drinkwater, D. T. An anatomically deroved method for the anthropometric estimation of human body composition. Ph.D. Thesis. British Columbia, Simon Fraser University, 1984.

Gorostiaga, E. Fútbol Femenino: bases fisiológicas, evaluación y prescripción del entrenamiento físico. Instituto Navarro de Deporte y Juventud. Navarra, Cuadernos Técnicos del Deporte, 2002. pp.16-56.

Henríquez-Olguín, C.; Báez, E.; Ramírez-Campillo, R. \& Cañas, R. Somatotype profile of professional male soccer chilean players. Int. J. Morphol., 31(1):225-30, 2013.

Hoare, D. G. \& Warr, C. R. Talent identification and women's soccer: an Australian experience. J. Sports Sci., 18(9):751-8, 2000.

Juric, I.; Sporis, G. \& Mihacic, M. Analysis of morphological features and played team positions in elite female soccer players. Proceedings of the VIth World Congress on Science and Football. Antalya, Plenum Press, 2007.

Kerr, D. A. An anthropometric method for fractionation of skin, adipose, bone, muscle and residual tissue masses, in males and females age 6 to 77 years. Thesis Master of Science in Kinesiology. British Columbia, Simon Fraser University, 1988.

Levandosky, G.; Cardozo, F. L.; Cieslak, F. \& Cardoso, A. S. Somatotype profile, anthropometric variables, physical aptitude and motor behavior of juvenile athletes of female futsal time from Ponta Grossa (Paraná-Brazil). Fit. Perf. J., 6(3):162-6, 2007.

Norton, K. \& Olds, T. Anthropometrica. Sydney, University of New South Wales Press, 1996.

Queiroga, M. R.; Ferreira, S. A.; Pereira, G. \& Kokubun, E. Somatotipo como indicador de desempenho em atletas de futsal feminino. Rev. Bras. Cineantropom. Desempenho. Hum., 10(1):56-61, 2008.

Shephard, K. \& Astrand, P. La resistencia en el deporte. Barcelona, Paidotribo, 1998.

Tejedor, M. \& Torés, K. Fútbol Femenino: Situación actual en entrenamiento y competición. Madrid, V Jornadas Técnicas de Preparadores Físicos de Fútbol Españoles, 2003.

Todd, M. K.; Scott, D. \& Chisnall, P. J. Fitness characteristics of English female soccer players: an analysis by position and playing standard. En: Spinks, W.; Reilly, T. \& Murphy, A. Science and Football IV. Londres, Routledge, 2002. pp.374-81.

Willmore, J. H. \& Costill, D. L. Fisiología del Esfuerzo y del Deporte. Barcelona, Paidotribo, 2004.

Dirección para Correspondencia:

Prof. Fernando Barraza Gómez

Escuela de Educación

Carrera Educación Física

Universidad Viña del Mar

Viña del Mar

CHILE

Email: fbarrazauvm@hotmail.com

Recibido : $14-03-2015$

Aceptado: 28-07-2015 